\title{
MINI-REVIEW
}

\section{Polycystic Ovary Syndrome and Risk of Endometrial Cancer: a Mini-Review}

\author{
Aytekin Tokmak, Mahmut Kuntay Kokanali*, Ali Irfan Guzel, Aydan Kara, \\ Hasan Onur Topcu, Sabri Cavkaytar
}

\begin{abstract}
The polycystic ovary syndrome is the most common endocrinological disorder of reproductive age women with a prevalence of 5 to $8 \%$. The most common diagnostic criteria used for polycystic ovary syndrome are oligo- or an-ovulation, clinical and/ or biochemical signs of hyperandrogenism and polycystic ovaries. Hyperandrogenism results in increased estrogen levels and lack of cyclic progesterone due to anovulation and persistent stimulation of the endometrium may lead to endometrial hyperplasia or adenocarcinoma development. In this mini review, we aimed to evaluate the possible relationship between polycystic ovary syndrome and endometrial cancer.
\end{abstract}

Keywords: Polycystic ovary syndrome - risk - endometrial cancer

Asian Pac J Cancer Prev, 15 (17), 7011-7014

\section{Introduction}

Polycystic ovary syndrome (PCOS) is a chronic, heterogeneous and multisystem endocrinological disorder that affects 5 to $8 \%$ of reproductive age women (Barry et al., 2014). Historically, there are some consensuses on PCOS diagnosis. These are by National Institute of Child Health and Human Development (NICHD) in 1990, the European Society for Human Reproduction and Embryology and American Society for Reproductive Medicine (ESHRE/ ASRM or Rotterdam) in 2003, and Androgen Excess Society (AES) in 2006. All of these definitions indicate the important role of hyperandrogenism in PCOS (Nandi et al., 2014). Obesity, type II diabetes mellitus and impaired glucose intolerance (IGI) are clinical features with a high prevalence in PCOS patients. Metabolic syndrome which includes dyslipidemia, obesity, IGI and hypertension also may be seen more common in these patients (Broekmans and Fauser, 2006; Fauser et al., 2012). PCOS is also related with increased estrogen levels and reduced apoptosis (Shafiee et al., 2013). Haulo et al. (2012) demonstrated that women with PCOS are three times more under risk of endometrial cancer (EC).

$\mathrm{EC}$ is the fourth common cancer among women with a ratio of 6 to $9 \%$ (Rowlands et al., 2011) and Arab et al. (2014) reported that EC revealed the highest age specific incidence after 59 years old among the gynecological cancers. EC has two types that type I is well differentiated and estrogen dependent form with a ratio of $75-85 \%$ of all EC and type II estrogen independent and clinically aggressive form (Di Cristofano et al., 2007; Lee et al., 2012; Binesh et al., 2014). Previous studies reported the increased risk of endometrial precancerous lesions and EC in PCOS patients (Hanprasertpong et al., 2008; Fearnley et al., 2010; Haoula et al., 2012).

In this mini review, we aimed to evaluate the possible association between PCOS and risk of EC under the headings of subgroups including; hyperandrogenism, hyperestrogenism and progesterone deficiency, insulin resistance (IR) and obesity.

\section{PCOS and Hyperandrogenism}

Hyperandrogenism is the most common diagnostic component of PCOS. It may be assessed by clinical features, biochemical indices, or both (Teede el al., 2010). This condition is related to the chronic anovulation. In adult women with PCOS, luteinizing hormone (LH) secretion is characterized by increased pulse frequency and amplitude, elevated 24-hour mean serum concentrations, and greater responses to gonadotropine releasing hormone $(\mathrm{GnRH})$ compared to those found in normal women. In humans, progressive increases in the frequency of GnRH stimulation have resulted in corresponding increases in the rate of LH release as well as elevated basal concentrations. This altered mechanism allows to pituitary LH hyper secretion resulting in excessive theca cell stimulation and also PCOS patients are tend to be genetically impaired theca cell functional defect. Consequently hyperandrogenism develops (Livadas et al., 2014). The alteration of enzymes functions involved in the synthesis of testosterone, such as $3 \beta$-hydroxysteroid dehydrogenase, CYP17, and CYP11A is also related to be responsible from the hyperandrogenism in PCOS (Escobar-Morreale et al., 2005). This increased level of androgens collaborates in the development of anovulation 
(Franks et al., 2000). Peripheral conversion of androgens to estrogen leads to the chronic exposure of endometrium to estrogen and may result in endometrial hyperplasia and EC (Kaaks et al., 2002).

Hyperandrogenism, itself, also is a common finding in endometrial cancer. It has been shown that androgen receptor and 5a-reductases are present in human endometrium. In some PCOS women, with over expression of endometrial androgen receptors disordered androgen action within the endometrium may result in EC (Dumesic and Lobo, 2013).

\section{PCOS, Hyperestrogenism and Progesterone Deficiency}

The majority of ECs occur due to the unopposed estrogen environment. In this situation, that the excess of estrogen stimulation is not sufficiently counterbalanced by progesterone results in promoted mitogenesis, atypical hyperplasia, and the transition to malignant adenocarcinoma (Wang et al., 2014). The presence of increased endometrial proliferation rates at follicular phase of menstrual cycle, during which progestin levels are low, whereas estradiol levels are at normal premenopausal concentrations and increased endometrial cancer risk among women using exogenous estrogens without progestins are two important signs that verify this relation (Kaaks et al, 2002)

Progesterone reduces estrogenic activity in the endometrium by enhancing the local synthesis of $17 \beta$-hydroxysteroid dehydrogenase and estrogen sulfotransferase (Sinreih et al., 2013). These enzymes play significant role in the conversion of estradiol into the less potent estrogen estrone, and into estrogen sulfates that are rapidly excreted from cells and from the body.

Mostly, the proliferative effects of estradiol on endometrial tissue are mediated by an increase in the local production, especially by stromal tissue, of insulin-like growth factor-1 (IGF-I) (Eritja et al., 2013). Progesterone provides the key stimulus for endometrial gene expression and synthesis of insulin-like growth factor binding protein-1 (IGFBP-1), which inhibits IGF-1 action in endometrial tissue (Kim and Chapman-Davis, 2010; Yang et al., 2011).

In patients with PCOS the increased estrogen levels and reported resistance of endometrium to progesterone stimulates the hyperplasic effects of estrogen to endometrium and risk of EC (Shen et al., 2008).

\section{PCOS, Insulin Resistance and Obesity}

Although the demonstration of IR is not necessary to diagnose PCOS, it is well established that hyperinsulinemic IR has a great importance in PCOS. The prevalence of IR in PCOS ranges from 50\%-70\% and occurs independently of obesity. However, obesity has an additive effect on IR in women with PCOS (Sirmans and Pate, 2014). IR is a condition in which target tissues become less sensitivity to insulin, results in elevated blood insulin levels. To overcome this, $\beta$ cells produce more insulin and compensatory hyperinsulinemia occurs. Consequently, increased insulin levels may induce cancer formation including breast cancer, colorectal cancer, prostate, pancreatic cancer and EC by several direct and indirect pathways (Mu et al., 2012).

Insulin directly promotes endometrial cell proliferation and activates protein-kinase enzyme systems [phosphatidylinositide-3-kinases (PI3K) and mitogen-activated-protein kinase (MAPK)] which have mitogenic and anti-apoptotic effects that contribute significantly to cellular transformation and development of EC (Subramaniam et al., 2013). Moreover, the interaction between insulin, estrogen and IGF-1 also contributes to the development of EC. Since the insulin receptors and IGF-1 receptors are partially homologoes, insulin can bind to IGF-1 receptor to activate protein-kinase signaling pathways (Janssen and Varewijck, 2014). As these pathways activated, they can stimulate transcriptional activation function-1 (TAF-1) of estrogen receptor, which regulates cell growth and division (Kato et al., 2000) Increased insulin levels can also inhibit IGFBP-1 mRNA and protein expression in endometrial stromal cells (Lathi et al., 2005) which leads elevated levels of free IGF-1. IGF-1 is a potent mitogen and survival factor that can promote the development of EC (Mu et al., 2012).

In the indirect mechanism, hyperinsulinemia can stimulate theca cell androgen activity, elevate serum free testosterone levels through decreased hepatic sex hormone-binding globulin (SHBG) production, amplify LH- and IGF-I-stimulated androgen production, and enhance serum IGF-I bioactivity through suppressed IGF-binding protein production which in turn promote the development of EC (Goodarzi et al., 2011).

Obesity is common in PCOS, which is a strong risk factor for EC (Crosbie et al., 2012). Insulin can behave as a growth factor in adipose tissue. It enlarges adipocytes as well as increases the number of adipocytes that causes obesity. In adipose tissue, estrogens can be produced by stromal cells or aromatization of adrenal androgens. In addition, the level of sex hormone binding globulin is decreased in obese women. These hormonal changes in obese women with PCOS contribute to increased activity of free estrogens that amplifies estrogenic activity in the endometrium and act in an additive manner to promote the development of EC. As the obesity and overweight are clearly correlated to insulin resistance and hyperinsulinemia, briefly the increased risk of EC in obese women is likely to be associated with steroid hormone disregulation during the insulin-resistant state (Villavicencio et al., 2012; De Pergola and Silvestris, 2013; Li and Shao, 2014).

\section{Conclusions}

In conclusion, Women with PCOS have a nearly 3 times increased risk for developing endometrial cancer, the association between PCOS and endometrial cancer involves prolonged endometrial exposure to unopposed estrogen by cyclic progesterone due to anovulation. Hyperandrogenism can cause EC by peripheral conversion to estrogen and through endometrial androgen receptors as well. PCOS and obesity are linked through their common 
symptom of IR. IR is an important potential risk factor of EC. High levels of insulin induced by IR have direct and indirect effects for the development of EC. Insulin directly stimulates endometrial cell proliferation and activates mitogenic and anti-apoptotic signaling systems in endometrium. And also, the network among insulin, estrogen and IGF-1 enhances the risk of the development of EC. Indirectly, insulin causes changes in sex hormone levels, including increases in the levels of estrogen that promotes the risk of EC. In addition, obesity, itself is a serious health problem and well-established risk factor for EC. So it is imperative women who have PCOS should be made aware about the increased risk of EC and also primary interventions, such as lifestyle changes. Shortly, the mechanisms underlying how PCOS increases the risk of EC is becoming better understood, thus it is obvious that several behavioral and pharmaceutical therapies will be developed for the prevention of both primary and recurrent disease in the future.

\section{References}

Arab M, Noghabaei G, Kazemi SN (2014). Comparison of crude and age-specific incidence rates of breast, ovary, endometrium and cervix cancers in Iran, 2005. Asian Pac J Cancer Prev, 15, 2461-4.

Barry JA, Azizia MM, Hardiman PJ (2014). Risk of endometrial, ovarian and breast cancer in women with polycystic ovary syndrome: a systematic review and meta-analysis. Hum Reprod Update, [Epub ahead of print].

Binesh F, Akhavan A, Behniafard N, Jalilian S (2014). Endometrial adenocarcinoma: clinicopathologic and survival characteristics in Yazd, Iran. Asian Pac J Cancer Prev, 15, 2797-801.

Broekmans FJ, Fauser BC (2006). Diagnostic criteria for polycystic ovarian syndrome. Endocrine, 30, 3-11.

Clarke CL., Adams JB, Wren BG (1982). Induction of estrogen sulfotransferase in the human endometrium by progesterone in organ culture. J Clin Endocrinol Metab, 55, 70-5.

Crosbie EJ, Roberts C, Qian W, et al (2012). Body mass index does not influence post-treatment survival in early stage endometrial cancer: results from the MRC ASTEC trial. Eur J Cancer, 48, 853-64.

De Pergola G, Silvestris F (2013). Obesity as a major risk factor for cancer. J Obes, 2013, 291546.

Di Cristofano A, Ellenson LH (2007). Endometrial carcinoma. Annu Rev Pathol, 2, 57-85.

Dumesic DA, Lobo RA (2013). Cancer risk and PCOS. Steroids. 78, 782-5.

Eritja N, Mirantes C, Llobet D, et al (2013). Long-term estradiol exposure is a direct mitogen for insulin/EGF-primed endometrial cells and drives PTEN loss-induced hyperplasic growth. Am J Pathol.183, 277-87.

Escobar-Morreale HF, Luque-Ramirez M, San Millan JL (2005). The molecular-genetic basis of functional hyperandrogenism and the polycystic ovary syndrome. Endocr Rev, 26, 251-82.

Fauser BC, Tarlatzis BC, Rebar RW, et al (2012). Consensus on women's health aspects of polycystic ovary syndrome (PCOS): the Amsterdam ESHRE/ASRM-Sponsored 3rd PCOS Consensus Workshop Group. Fertil Steri, 97, 28-38.

Fearnley EJ, Marquart L, Spurdle AB, Weinstein P, Webb PM (2010). Polycystic ovary syndrome increases the risk of endometrial cancer in women aged less than 50 years: an Australian case-control study. Cancer Causes Control, 21, 2303-8.
Franks S, Mason H, Willis D (2000). Follicular dynamics in the polycystic ovary syndrome. Mol Cell Endocrinol, 163, 49-52.

Goodarzi M, Dumesic DA, Chazenbalk G, Azziz R (2011). Polycystic ovary syndrome: etiology, pathogenesis and diagnosis. Nat Rev Endocrinol, 7, 219-31.

Hanprasertpong J, Sakolprakraikij S, Geater A (2008). Endometrial cancer in Thai women aged 45 years or younger. Asian Pac J Cancer Prev, 9, 58-62.

Haoula Z, Salman M, Atiomo W (2012). Evaluating the association between endometrial cancer and polycystic ovary syndrome. Hum Reprod, 27, 1327-31.

Janssen JA, Varewijck AJ (2014). Insulin analogs and cancer: a note of caution. Front Endocrinol, 5, 79.

Kaaks R, Lukanova A, Kurzer MS (2002). Obesity, endogenous hormones, and endometrial cancer risk: a synthetic review. Cancer Epidemiol Biomarkers Prev. 11, 1531-43.

Kato S, Masuhiro Y, Watanabe M, et al (2000). Molecular mechanismof a cross-talk between oestrogen and growth factor signalling pathways. Genes Cells, 5, 593-601.

Kim JJ, Chapman-Davis E (2010). Role of progesterone in endometrial cancer. Semin Reprod Med, 28, 81-90.

Kitawaki J, Yamamoto T, Okada H (1998). Induction of estradiol dehydrogenase activity in human uterine endometrium by synthetic steroids. J Endocrinol Investig, 11, 351-54.

Lathi RB, Hess AP, Tulac S, et al (2005). Dose-dependent insulin regulation of insulin-like growth factor binding protein-1 in human endometrial stromal cells is mediated by distinct signaling pathways. J Clin Endocrinol Metab, 90, 1599-606.

Lee WL, Lee FK, Su WH et al (2012). Hormone therapy for younger patients with endometrial cancer. Taiwan J Obstet Gynecol, 51, 495-505.

Li X, Shao R (2014). PCOS and obesity: insulin resistance might be a common etiology for the development of type I endometrial carcinoma. Am J Cancer Res, 15, 73-9.

Livadas S, Pappas C, Karachalios A, et al (2014). Prevalence and impact of hyperandrogenemia in 1,218 women with polycystic ovary syndrome. Endocrine, [Epub ahead of print].

Mu N, Zhu Y, Wang Y, Zhang H, Xue F (2012). Insulin resistance: a significant risk factor of endometrial cancer. Gynecol Oncol, 125, 751-7.

Nandi A, Chen Z, Patel R, Poretsky L (2014). Polycystic ovary syndrome. Endocrinol Metab Clin North Am, 43, 123-47.

Quezada S, Avellaira C, Johnson MC, et al (2006). Evaluation of steroid receptors, coregulators, andmolecules associatedwith uterine receptivity in secretory endometria from untreated women with polycystic ovary syndrome. Fertil Steril, $\mathbf{8 5}$, 1017-26.

Rowlands IJ, Weinstein P, Nagle CM, et al (2011). Season of birth and risk of endometrial cancer. Asian Pac J Cancer Prev, 12, 1193-6.

Shafiee MN, Chapman C, Barrett D, Atiomo W (2013). Reviewing the molecular mechanisms which increase endometrial cancer risk in women with polycystic ovarian syndrome: time for paradigm shift? Gynecol Oncol, 131, 489-92.

Shen ZQ, Zhu HT, Lin JF (2008). Reverse of progestin-resistant atypical endometrial hyperplasia by metformin and oral contraceptives. Obstet Gynecol, 112, 465-7.

Sinreih M, Hevir N, Rizner TL (2013). Altered expression of genes involved in progesterone biosynthesis, metabolism and action in endometrial cancer. Chem Biol Interact, 202, 210-7.

Sirmans SM, Pate KA (2013). Epidemiology, diagnosis, and management of polycystic ovary syndrome. Clin Epidemiol, 6, 1-13.

Subramaniam KS, Tham ST, Mohamed Z, et al (2013). Cancer- 
Aytekin Tokmak et al

associated fibroblasts promote proliferation of endometrial cancer cells. PLoS One, 8, 68923.

Teede H, Deeks A, Moran L (2010). Polycystic ovary syndrome: a complex condition with psychological, reproductive and metabolic manifestations that impacts on health across the lifespan. BMC Med, 8, 41.

Wang HL, Liu MM, Ma X, et al (2014). Expression and effects of JMJD2A histone demethylase in endometrial carcinoma. Asian Pac J Cancer Prev, 15, 3051-6.

Yang S, Thiel KW, Leslie KK (2011). Progesterone: the ultimate endometrial tumor suppressor. Trends Endocrinol Metab. 22, 145-52. 